\title{
The Constraint on FCNC Coupling of the Top Quark with a Gluon from ep - Collisions
}

\author{
A.A. Ashimova 1 \\ Moscow State University, 119992 Moscow, Russia \\ S.R. Slabospitsky 2 \\ State Research Center \\ Institute for High Energy Physics, \\ Protvino, Moscow Region 142281, Russia
}

\begin{abstract}
Using the constraint on the single top production cross-section obtained at the HERA collider, $\sigma(e p \rightarrow$ et $X)$, we evaluate an upper limit on coupling constant of the anomalous top quark interaction with a gluon via flavor-changing neutral current: $\left|\kappa_{\text {tgq }} / \Lambda\right| \lesssim$ $0.4 \mathrm{TeV}^{-1}, \mathrm{BR}(t \rightarrow g q)<13 \%$
\end{abstract}

\footnotetext{
${ }^{1}$ E-mail: ashimova_aa@mail.ru

${ }^{2}$ E-mail: Sergey.Slabospitsky@ihep.ru
} 


\section{Introduction}

In different extensions of the Standard Model (SM) the anomalous interactions can lead to significant modification of the top quark production mechanisms and appearance of the top rare decays [1]. Thereby, the precision measurements of the top quark properties and its production mechanisms provide a possibility to obtain an information on new physics beyond the SM. Of special interest it is the study of the top quark anomalous interactions via Flavour-Changing Neutral Currents (FCNC).

In the SM there are no direct FCNC transitions $t \rightarrow u(c)$. Only "loop" contributions can make them possible. As a result such processes are strongly suppressed within the SM: $\mathrm{BR}(t \rightarrow(\gamma, g, Z)+u(c))<\mathcal{O}\left(10^{-10}\right)$ [2]. However, various extensions of the SM could lead to a huge enhancement of FCNC processes [3, 4, 15].

Search for the anomalous FCNC interactions of the top quark were performed at the Tevatron [6], HERA [7, 8, 9], and LEP-2 [10] colliders. The present constraints on the top quark anomalous couplings in terms of branching ratio (BR) are presented in the Table below.

Table 1: The present constraints on top quark anomalous couplings

\begin{tabular}{|c|c|c|}
\hline decay & BR & collider \\
\hline \hline$t \rightarrow q \gamma$ & $<0.7 \%$ & HERA [8, 9] \\
$t \rightarrow q Z$ & $<7 \%$ & LEP 2 [10] \\
$t \rightarrow q g$ & $<8 \%$ & Tevatron + th. [1] \\
\hline
\end{tabular}

Search for the top production at HERA collider in ep-collisions,

$$
e p \rightarrow e t X
$$

was done by the H1 [8] and ZEUS [9] collaborations at the center-mass energy of $\sqrt{s}=$ $318 \mathrm{GeV}$. Obviously that only single top production is possible at this energy. In this case the $\mathrm{SM}$ dominant process is $e p \rightarrow \nu t \bar{b} X$. However, the production cross-section for this process at HERA energy is very small $(\sigma(e p \rightarrow t X)<1 \mathrm{fb}[12])$ and far beyond an experimental observation. On the other hand the anomalous FCNC process could contribute to the single top production at HERA energies. Therefore, a study of such reaction at HERA provide a good possibility to put a constraint on FCNC interactions.

The both H1 and ZEUS collaborations searched for the top quark production in reaction (11). No evidence of the top production was found. The resulted constraint on the single top production cross-section is given below [8, 9]:

$$
\sigma(e p \rightarrow \text { et } X)<0.225 \mathrm{pb} \quad(\text { at } 95 \% \mathrm{CL})
$$

The conventional interpretation of this data assumes that the top quark anomalous FCNC interactions could occur with a photon or $Z$-boson only (see Fig. 1). As a result from 
the experimental upper limit (2) the constraint on the value of anomalous coupling was evaluated [8, 9]. In terms of BR this constraint is as follows:

$$
\mathrm{BR}(t \rightarrow q \gamma)<0.7 \%
$$

In the present article we consider an additional FCNC process that contribute to the single top-quark production at HERA energies. Indeed, in ep-collisions the single top could be produced also due to anomalous interaction with a gluon, i.e. $\operatorname{tgq}$ vertex, as it is shown on fig. 2. Thereby using the upper limit on the production cross-section (2) we evaluate a constraint on anomalous FCNC coupling of the top quark with a gluon. Therein lies the basic idea of our analysis.

\section{Phenomenological Lagrangian for FCNC}

We do not know which type of new physics will be responsible for a future deviation from the SM predictions. However, the top quark couplings can be parameterized in a model independent way by an effective Lagrangian [1] (see also [13] for the first discussion of the tc g-operator).

In our article we consider the top quark anomalous interactions with gluon and photon. However for the completeness we also present here the expression for the $t$-quark FCNC interaction with $Z$-boson. Actually the width of $t \rightarrow Z q$ decay is used in the Section 4. Thus the effective phenomenological Lagrangian have a form as follows:

$$
\begin{aligned}
\mathcal{L}= & -2 g_{s} \sum \frac{\kappa_{g}}{\Lambda} \bar{t} \sigma_{\mu \nu} T^{a}\left(g_{L} P_{L}+g_{R} P_{R}\right) u q^{\nu} G_{a}^{\mu} \\
& -2 e \sum \frac{\kappa_{\gamma}}{\Lambda} \bar{t} \sigma_{\mu \nu}\left(\gamma_{L} P_{L}+\gamma_{R} P_{R}\right) u q^{\nu} A^{\mu} \\
& -\frac{e}{\sin 2 \vartheta_{W}} \sum \kappa_{Z} \bar{t} \gamma^{\mu}\left(z_{L} P_{L}+z_{R} P_{R}\right) u Z_{\mu}+\text { h.c. }
\end{aligned}
$$

where $t^{a}$ are the Gell-Mann matrices satisfying $\operatorname{Tr}\left(t^{a} t^{b}\right)=\delta^{a b} / 2 ; \kappa_{g}$ and $\kappa_{\gamma}$ define the strength of the FCNC with a gluon and a photon respectively (real and positive values); $\Lambda=1 \mathrm{TeV}$ is a new physics cutoff; $e$ is the electric charge; $\vartheta_{W}$ is the Weinberg angle; $\sigma^{\mu \nu}=\frac{1}{2}\left(\gamma^{\mu} \gamma^{\nu}-\gamma^{\nu} \gamma^{\mu}\right)$; $P_{R, L}=\frac{1}{2}\left(1 \pm \gamma^{5}\right) ; g_{L}^{2}+g_{R}^{2}=\gamma_{L}^{2}+\gamma_{R}^{2}=1 ; q^{\nu}$ is the momentum of corresponding gauge boson.

The top-quark decay widths into one of the light quarks ( $u$ or $c$ ) with a gluon, photon and $Z$-boson have the form:

$$
\begin{gathered}
\Gamma(t \rightarrow g q)=\frac{4}{3} \alpha_{s} m_{t}^{3}\left(\frac{\kappa_{g}}{\Lambda}\right)^{2} \\
\Gamma(t \rightarrow \gamma q)=\alpha m_{t}^{3}\left(\frac{\kappa_{\gamma}}{\Lambda}\right)^{2} \\
\Gamma(t \rightarrow Z q)=\frac{\alpha \kappa_{Z}^{2} m_{t}^{3}}{8 \sin ^{2} 2 \vartheta_{W} M_{Z}^{2}}\left(1-\frac{M_{Z}^{2}}{m_{t}^{2}}\right)^{2}\left(1+2 \frac{M_{Z}^{2}}{m_{t}^{2}}\right)
\end{gathered}
$$




\section{The matrix elements squared}

Firstly, we re-calculate the matrix element squared for $2 \rightarrow 2$ process: $e u \rightarrow$ et (see the digram on Fig. 1). This expression is used in our analysis. The explicit form [15] is given below:

$$
\begin{aligned}
|M|^{2} & =4 e^{4}\left(\frac{1}{Q^{2}}\right)^{2}\left(\frac{\kappa_{\gamma}}{\Lambda}\right)^{2} \times \\
& \left\{2 m_{e}^{2} \hat{t}(2 \hat{s}+\hat{t})-2 m_{e}^{2} m_{t}^{4}-\hat{t}\left[2 \hat{s}\left(\hat{s}-m_{t}^{2}\right)+m_{t}^{4}\right]-\hat{t}^{2}\left(2 \hat{s}-m_{t}^{2}\right)\right\}
\end{aligned}
$$

where $\hat{s}$ and $\hat{t}$ are usual Mandelstam variables, $Q^{2}=|\hat{t}|$ is a photon transferred momentum squared, $m_{e}$ and $m_{t}$ are the masses of the electron and $t$-quark. Note, that we keep non-zero electron mass, because this is essential for the further analysis.

One can obtain an expression for the production cross-section of the hard $2 \rightarrow 2$ process (corresponding to matrix element squared (8) ) by the integration on $Q^{2}$ from $Q_{\min }^{2}$ to $Q_{\max }^{2}$ :

$$
\begin{aligned}
\hat{\sigma}\left(Q_{\min }^{2}\right) & =C\left[B \ln \left(\frac{Q_{\max }^{2}}{Q_{\min }^{2}}\right)-A\left(Q_{\max }^{2}-Q_{\min }^{2}\right)\right] \\
& -2 C m_{e}^{2}\left[2 \hat{s} \ln \left(\frac{Q_{\max }^{2}}{Q_{\min }^{2}}\right)-\left(Q_{\max }^{2}-Q_{\min }^{2}\right)\right] \\
& +2 C m_{e}^{2} m_{t}^{4}\left(\frac{1}{Q_{\min }^{2}}-\frac{1}{Q_{\max }^{2}}\right),
\end{aligned}
$$

where

$$
C=\frac{\pi e^{4}}{2 \hat{s}\left(\hat{s}-m_{e}^{2}\right)}\left(\frac{\kappa_{\gamma}}{\Lambda}\right)^{2}, \quad A=2 \hat{s}-m_{t}^{2}, \quad B=2 \hat{s}\left(\hat{s}-m_{t}^{2}\right)+m_{t}^{4}
$$

The single top production in $e p$-collisions due to anomalous FCNC interaction with a gluon is described by two subprocesses with a gluon or $u(c)$-quark from the initial proton (see the diagrams on Fig. 2):

$$
\begin{aligned}
& e g \rightarrow e t q, \quad q=u \text { or } c \\
& e q \rightarrow e t g, \quad q=u \text { or } c
\end{aligned}
$$

Note, that the matrix elements squared for these two processes are related to each other by crossing. Therefore, we present an exact expression for $|M|^{2}$ for the first subprocess (10):

$$
|T|^{2}=\left|T_{1}+T_{2}\right|^{2}
$$

The exact expressions are given below:

$$
\begin{aligned}
\left|T_{1}\right|^{2}= & A_{0} A_{1}^{2}\left(k p_{2}\right)\left\{\left(f_{1} l_{1}\right)\left(p_{1} l_{2}\right)+\left(f_{1} l_{2}\right)\left(p_{1} l_{1}\right)-\left(p_{1} f_{1}\right)\left[\left(l_{1} l_{2}\right)+\frac{q^{2}}{2}\right]\right\} \\
& -2 A_{0} A_{1}^{2} m_{t}^{2}\left(k p_{2}\right)\left[\left(l_{1} l_{2}\right)+q^{2}\right] \\
\left|T_{2}\right|^{2}= & A_{0} A_{2}^{2}\left(k p_{1}\right)\left\{\left(f_{2} l_{1}\right)\left(p_{2} l_{2}\right)+\left(f_{2} l_{2}\right)\left(p_{2} l_{1}\right)-\left(p_{2} f_{2}\right)\left[\left(l_{1} l_{2}\right)+\frac{q^{2}}{2}\right]\right\} ;
\end{aligned}
$$




$$
\begin{aligned}
\frac{T_{1}^{+} T_{2}+T_{2}^{+} T_{1}}{A_{0} A_{1} A_{2}} & =4\left(p_{1} l_{1}\right)\left(p_{1} l_{2}\right)\left(k p_{2}\right)^{2}+4\left(p_{2} l_{1}\right)\left(p_{2} l_{2}\right)\left(k p_{1}\right)^{2}+4\left(k l_{1}\right)\left(k l_{2}\right)\left(p_{1} p_{2}\right)^{2} \\
& -2\left(p_{1} p_{2}\right)\left\{2\left(f_{3} l_{1}\right)\left(k l_{2}\right)+2\left(f_{3} l_{2}\right)\left(k l_{1}\right)-\left(k f_{3}\right)\left(l_{1} l_{2}\right)\right\} \\
& +2 m_{t}^{2}\left(k p_{2}\right)\left\{\left(p_{2} l_{1}\right)\left(k l_{2}\right)+\left(p_{2} l_{2}\right)\left(k l_{1}\right)-\left(k p_{2}\right)\left(l_{1} l_{2}\right\}\right. \\
& -m_{t}^{2}\left\{2\left(f_{3} l_{1}\right)\left(k l_{2}\right)+2\left(f_{3} l_{2}\right)\left(k l_{1}\right)-\left(k f_{3}\right) q^{2}\right\} \\
& +2 m_{t}^{2}\left(k l_{1}\right)\left(k l_{2}\right)\left(p_{1} p_{2}\right) ;
\end{aligned}
$$

where

$$
\begin{aligned}
& A_{0}=64\left[\frac{4}{3} \frac{\kappa_{g}}{\Lambda} \alpha_{s} e^{2}\right]^{2}, A_{1}=-\frac{1}{2\left(k p_{2}\right)+m_{t}^{2}}, \quad A_{2}=-\frac{1}{2\left(k p_{1}\right)-m_{t}^{2}} \\
& f_{1}=2\left(k p_{2}\right) p_{2}+m_{t}^{2} k, f_{2}=2\left(k p_{1}\right) p_{1}-m_{t}^{2} k, f_{3}=\left(k p_{1}\right) p_{2}+\left(k p_{2}\right) p_{1}
\end{aligned}
$$

here $l_{1}\left(l_{2}\right)$ are 4-momentum of the initial (final) electron, $p_{1}$ and $p_{2}$ are 4 -momenta of the topand $\bar{u}$-quarks, and $k$ is the initial gluon momentum. The calculation of the color coefficients is trivial and they are included to $A_{0}$.

For numerical calculations the evaluated matrix elements squared are incorporated in the event generator TopReX [14].

\section{Kinematics of the considered processes}

The cross-section of the considered processes depends essentially on the kinematical limit of the gauge boson transferred momentum $Q_{\min }$. This limit is determined by the additional requirements on kinematics of the reaction and the detector parameters. For some reason the authors of the experimental paper [9] did not mention the limit on $Q_{\min }$ in an explicit form.

In order to calculate the theoretical value of the cross-section, $\sigma^{\text {th }}$, we must imply a correct limit on $Q_{\text {min }}$. This value can be estimated from the following considerations. The experimental analysis for $2 \rightarrow 2$ process was made under the assumption that single top is produced due to anomalous coupling with a photon. It is reasonable that the theoretical cross-section also depends on the $Q_{\min }$ kinematical limit and anomalous coupling with a photon. Therefore by using the value of the anomalous coupling one can compare theoretical cross-section with experimental one and can determine the unknown value of $Q_{m i n}^{2}$.

Thus we get the value of $Q_{\min }^{2}$ from the following condition:

$$
\sigma_{2 \rightarrow 2}^{\text {th }}(e p \rightarrow e t X)=\left(\frac{\kappa_{\gamma}}{\Lambda}\right)^{2} \times f_{2 \rightarrow 2}^{e p \rightarrow e t X}\left(Q_{\text {min }}^{2}\right) \leqslant \sigma^{e x p}=0.225 \mathrm{pb}
$$

Here $\sigma_{2 \rightarrow 2}^{\text {th }}$ is the total cross-section for $e p \rightarrow e t X$ process (with subprocess $e u \rightarrow e t$ ) calculated using the expression (9), $\sigma^{\exp }$ is the experimental limit on the single top quark production from the HERA data [9]. The upper limit on anomalous coupling of the top quark with a photon was taken from the same article [9]: $\kappa_{t u \gamma}^{H}<0.174$. 
Note that the authors of the experimental work [9] used different normalization for phenomenological Lagrangian:

$$
\mathcal{L}^{\mathcal{H}}=e e_{t} \bar{t} \frac{\sigma_{\mu \nu} q^{\nu}}{\Lambda^{H}} u \kappa_{t u \gamma}^{H},
$$

where $e$ is the electric charge, $e_{t}$ is the charge of up quarks and $\Lambda^{H}=m_{t}=175 \mathrm{GeV}$.

The relation between $\kappa_{t u \gamma}^{H}$ and $\kappa_{\gamma}$ used in our Lagrangian (44) looks as follows:

$$
\kappa_{\gamma}=\frac{e_{t}}{\sqrt{2}} \frac{\Lambda}{\Lambda^{H}} \frac{1}{\sqrt{\gamma_{L}^{2}+\gamma_{R}^{2}}} \kappa_{t u \gamma}^{H}
$$

Note that we take for the new physics cutoff $\Lambda=1 \mathrm{TeV}$ and by our convention $\gamma_{L}^{2}+\gamma_{R}^{2}=1$ (see Eq. (4)). As a result the upper limit on the anomalous coupling in terms of the Lagrangian (4) equals:

$$
\kappa_{\gamma}=0.468
$$

The single top production cross-section in $e p$-collsions was evaluated within the parton model:

$$
\sigma^{\text {th }}\left(e^{ \pm} p \rightarrow e^{ \pm} t X\right)=\int_{m_{t}^{2} / s}^{1} d x f\left(x, \mu^{2}\right) \hat{\sigma}\left(x, \mu^{2}\right),
$$

where $x$ is the part of the proton momentum carried away by a parton; $\hat{\sigma}\left(x, \mu^{2}\right)$ is the crosssection for the hard subprocess; $f\left(x, \mu^{2}\right)$ is the parton distribution function, and $\mu=m_{t}$ is the factorization scale.

Using (13) we obtain

$$
Q_{\text {min }}^{2} \geqslant 0.08 \mathrm{GeV}^{2}
$$

Further in our analysis of the single top production due to anomalous coupling with a gluon we implied the very same limit on $Q_{\min }^{2}(17)$.

\section{The constraint on FCNC coupling of the top-quark with a gluon}

In the present study we compare the experimental constraint (2) with the total top-quark production cross-section in $e p$-collisions (evaluated with the matrix elements squared (12) ).

$$
e p \rightarrow \operatorname{etf} X
$$

Thus the single top quark production in ep-collisions is possible not only via FCNC with a photon, but also due to anomalous interactions with a gluon. This idea gives us an opportunity to obtain the direct constraint on FCNC coupling of the top quark with a gluon from HERA experimental data (2).

In order to find the constraint on gluon coupling $\kappa_{g}$ we use the following relation: 


$$
\sigma^{t h}(e p \rightarrow \operatorname{etf} X) \times B R\left(t \rightarrow b W^{+}\right) \leqslant \sigma^{e x p}=0.225 \mathrm{pb}
$$

where

$$
\sigma_{2 \rightarrow 3}^{\mathrm{th}}(e p \rightarrow \operatorname{etf} X)=\left(\frac{\kappa_{g}}{\Lambda}\right)^{2} \times F_{2 \rightarrow 3}^{e p \rightarrow e t f X}\left(Q_{m i n}^{2}\right) .
$$

Here we take into account the possible modification of the branching ratio of the top decay in the SM mode $t \rightarrow b W^{+}$. As it was mentioned above the top quark anomalous interactions could lead to the appearance of additional top decay channels. So the probability of the SM top decay would change accordingly:

$$
B R\left(t \rightarrow b W^{+}\right)=\frac{\Gamma\left(t \rightarrow b W^{+}\right)}{\Gamma\left(t \rightarrow b W^{+}\right)+\Gamma(t \rightarrow Z q)+\Gamma(t \rightarrow g q)},
$$

To obtain an upper limit on the anomalous coupling with a gluon we assume the absence of the top-quark FCNC interaction with a photon, i.e. $\kappa_{t \gamma u, c}=0$. In addition the different cases of the top interactions with a gluon and $Z$-boson are explored:

1. $\kappa_{t Z u, c}=0, \kappa_{t g c}=0, \kappa_{t g u} \neq 0$,

the simplest case, i.e. gluon - top - $u$-quark interaction;

2. $\kappa_{t Z u, c}=0, \kappa_{t g c}=\kappa_{t g u} \neq 0$.

here the FCNC transitions with a gluon to $u$ - or $c$-quark are considered;

Two additional cases include the anomalous interaction with $Z$-boson:

3. $\kappa_{t Z u, c} \neq 0, \kappa_{t g c}=0, \kappa_{t g u} \neq 0$.

4. $\kappa_{t Z u, c} \neq 0, \kappa_{t g c}=\kappa_{t g u} \neq 0$.

According to all assumptions (1.-4.) the constraints on the top quark FCNC couplings with a gluon, $u$-, $c$-quarks and the corresponding BR of the anomalous top decays are calculated. The results of the analysis are presented in Table 2 ,

Table 2: The constraints on the top quark FCNC coupling with a gluon from the HERA data $\left(\kappa_{t \gamma q}=0\right)$

\begin{tabular}{|c|l|c|c|c|}
\hline & & $\kappa_{t g u} / \Lambda, \mathrm{TeV}^{-1}$ & $\kappa_{t g c} / \Lambda, \mathrm{TeV}^{-1}$ & $\mathrm{BR}(t \rightarrow g q)$ \\
\hline \hline 1 & $\kappa_{t Z q}=0, \kappa_{t g c}=0$ & 0.393 & - & $6.6 \%$ \\
\hline 2 & $\kappa_{t Z q}=0, \kappa_{t g u}=\kappa_{t g c}$ & 0.396 & 0.396 & $12.6 \%$ \\
\hline \hline 3 & $\kappa_{t Z q} \neq 0, \kappa_{t g c}=0$ & 0.408 & - & $6.2 \%$ \\
\hline 4 & $\kappa_{t Z q} \neq 0, \kappa_{t g u}=\kappa_{t g c}$ & 0.410 & 0.410 & $11.8 \%$ \\
\hline
\end{tabular}

The best constraint on FCNC coupling with a gluon from ep-collision is obtained supposing the interaction with an $u$-quark only. When the interaction with a charm quark is also 
taken into account the constraints on couplings do not change greatly but the BR increases almost twice. It is explained by the small contribution of the $c$-quarks to proton's PDF and appearance of two decay modes of the top-quark. One also can see that the contribution of $Z$-boson modifies the results slightly and can be neglected.

Finally the derived constraint on the anomalous coupling of the top FCNC interaction with a gluon and the corresponding BR are given below:

$$
\left|\kappa_{t g q} / \Lambda\right| \lesssim 0.4 \mathrm{TeV}^{-1}, \quad \mathrm{BR}(t \rightarrow g q)<13 \%
$$

\section{Conclusions}

Very recently at the Tevatron experiment [11] better constraint ( $3 \div 10$ times less) on the $\kappa_{g}$, the upper limit on the anomalous coupling of the top quark with a gluon, was obtained.

Nevertheless we wish to indicate that the HERA results for the single top production provides the way to put the constraints on the anomalous top-quark interactions not only with a photon and $Z$-boson, but also with a gluon!

Moreover, such analysis of ep-collision (e.g. HERA data) allows getting the limits on anomalous coupling of the top quark with a gluon and can be considered as an independent estimation of $\kappa_{g}$.

\section{Acknowledgments}

We are grateful to M. Mohammadi Najafabadi and O.V. Zenin for useful discussions. This work was supported in part by the Dynasty Foundation and by the Russian Foundation for Basic Research under Grant \# 08-02-91002-C.

\section{References}

[1] Beneke M. et al., "Top quark physics", in "Standard model physics (and more) at the LHC", G. Altarelli and M. L. Mangano eds., Geneva, Switzerland: CERN (2000) arXiv:hep-ph/0003033.

[2] Grzadkowski B., Gunion J.F., and Krawczyk P., Phys. Lett., B268, 106 (1991);

Eilam G., Hewett J.L, and Soni A., Phys. Rev. D44, 1473 (1991);

Luke M. and Savage M.J., Phys. Lett. B307, 387 (1993);

Couture G., Hamzaoui C., and Kønig H., Phys. Rev. D52, 1713 (1995).

[3] Atwood D., Reina L. and Soni A., Phys. Rev., D55, 3156 (1997) arXiv:hep-ph/9609279.

[4] Yang J.M., Young B.-L. and Zhang X., Phys. Rev., D58, 055001 (1998) arXiv:hep-ph/9705341. 
[5] F. del Aguila, J.A. Aguilar-Saavedra and R. Miquel, Phys. Rev. Lett. 82, 1628 (1999) arXiv:hep-ph/9808400|.

[6] Abe F. et al. [CDF Collaboration], Phys. Rev. Lett. 80, 2525 (1998).

[7] Wolf G., arXiv:hep-ex/0105055];

Alan A.T. and Senol A., Europhys. Lett. 59, 669 (2002) [arXiv:hep-ph/0202119];

Dannheim H. [H1 Collaboration], arXiv:hep-ex/0212004].

[8] Aktas A. et al. [H1 Collaboration], Eur. Phys. J. C33, 9 (2004) arXiv:hep-ex/0310032.

[9] Chekanov S. et al. [ZEUS Collaboration], Phys. Lett. B559, 153 (2003) arXiv:hep-ex/0302010].

[10] Obraztsov V.F., Slabospitsky S.R., and Yushchenko O.P., Phys. Lett. B426, 393 (1998) arXiv:hep-ph/9712394;

Heister A. et al. [ALEPH Collaboration], Phys. Lett. B543, 173 (2002) arXiv:hep-ex/0206070|;

Abreu P. et al. [DELPHI Collaboration], Phys. Lett. B446, 62 (1999) arXiv:hep-ex/9903072;

Abdallah J. et al. [DELPHI Collaboration], Phys. Lett. B590, 21 (2004) arXiv:hep-ex/0404014];

Achard P. et al. [L3 Collaboration], Phys. Lett. $\quad$ B549, 290 (2002) arXiv:hep-ex/0210041;

Abbiendi G. et al. [OPAL Collaboration], Phys. Lett. B521, 181 (2001) arXiv:hep-ex/0110009].

[11] Abazov V.M. et al. [D0 Collaboration] Phys. Lett. 99, 191802 (2007) arXiv:hep-ex/0702005.

[12] Stelzer T., Sullivan Z. and Willenbrock S., Phys. Rev. D 56, 5919 (1997) arXiv:hep-ph/9705398;

Moretti S. and Odagiri K., Phys. Rev. D 57, 3040 (1998) [arXiv:hep-ph/9709435].

[13] Malkawi E. and Tait T., Phys. Rev. D54 (1996) 5758 arXiv:hep-ph/9511337

Tait T. and Yuan C. P., Phys. Rev. D55 (1997) 7300 [arXiv:hep-ph/9611244.

[14] Slabospitsky S.R. and Sonnenschein L., "TopReX generator (version 3.25): Short manual", Comput. Phys. Commun. 148, 87 (2002) arXiv:hep-ph/0201292].

[15] Belyaev A. and Kidonakis N., Phys. Rev. D65, 037501 (2002). 


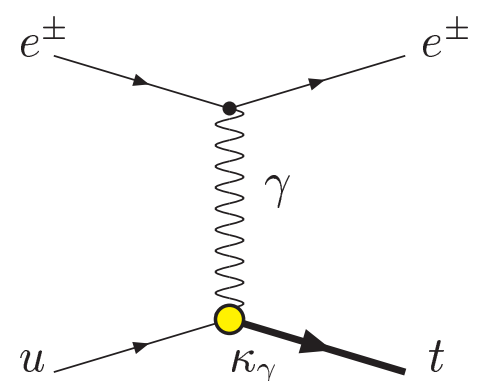

Figure 1: Single top production via FCNC coupling with photon (or Z-bozon)

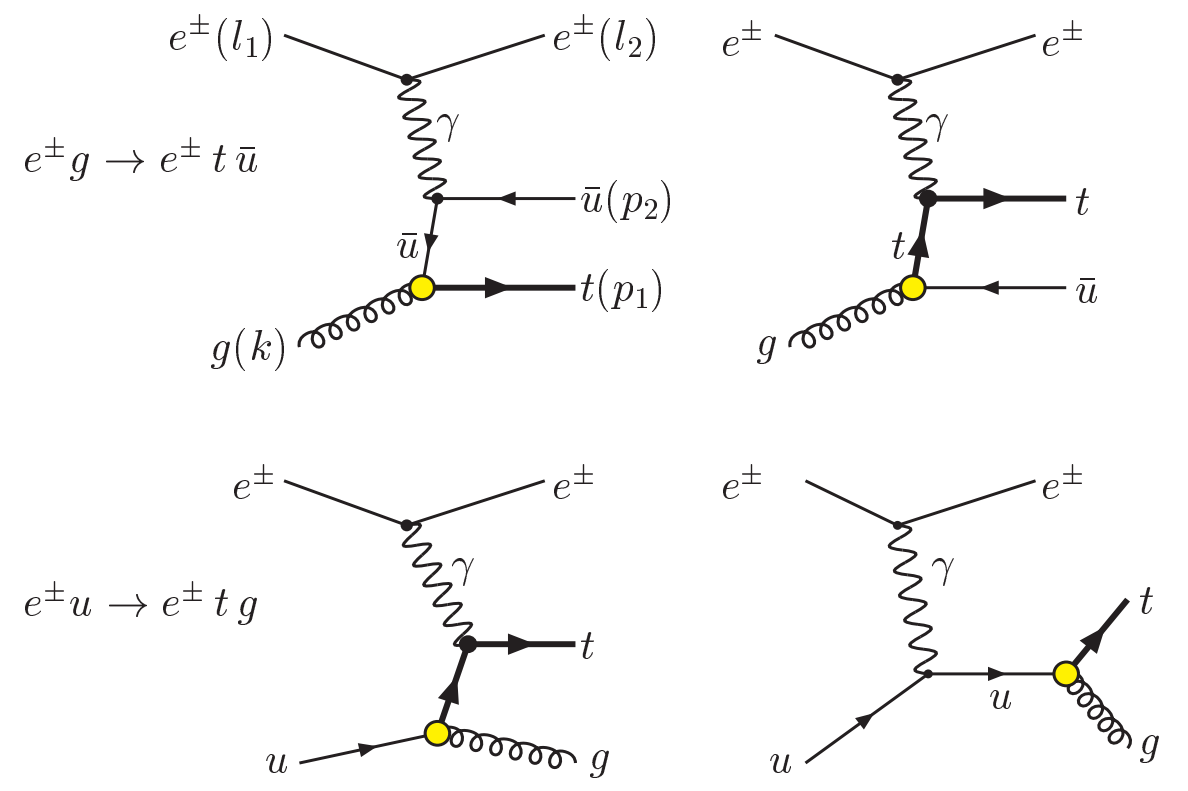

Figure 2: The Feynman diagrams of the single top production in ep-collisions due to FCNC with a gluon 